\title{
WEIGHTED NORLUND-EULER A-STATISTICAL CONVERGENCE FOR SEQUENCES OF POSITIVE LINEAR OPERATORS
}

\author{
Elida Hoxha ${ }^{1}$, Ekrem Aljimi $^{2}$ and Valdete Loku ${ }^{3}$
}

\begin{abstract}
We introduce the notion of weighted Norlund -Euler A-Statistical Convergence of a sequence, where A represents the nonnegative regular matrix. We also prove the Korovkin approximation theorem by using the notion of weighted Norlund-Euler A-statistical convergence. Further, we give a rate of weighted Norlund-Euler A-statistical convergence.
\end{abstract}

\section{BaCkground, Notations And Preliminaries}

Suppose that $E \subseteq N=\{1,2, \ldots\}$ and $E_{n}=\{k \leq n: k \in E\}$. Then

$$
\delta(E)=\lim _{n \rightarrow \infty} \frac{1}{n}\left|E_{n}\right|
$$

is called the natural density of $E$ provided that the limit exist, where |.| represents the number of elements in the enclosed set.

The term "statistical convergence" was first presented by Fast [1] which is generalization of the concept of ordinary convergence. Actually, a root of the notion of statistical convergence can be detected by Zygmund [2] (also see [3]), where he used the term 'almost convergence' which turned out to be equivalent to the concept of statistical convergence. The notion of Fast was further investigated by Schoenberg [4], Salat [5], Fridy [6], and Conner [7].

The following notion is due to Fast [1]. A sequence $x=\left(x_{k}\right)$ is said to be statistically convergent to $L$ if $\delta\left(K_{\varepsilon}\right)=0$ for every $\varepsilon>0$, where

$$
K_{\varepsilon}=\left\{k \in N:\left|x_{k}-L\right| \geq \varepsilon\right\}
$$

equivalently,

$$
\lim _{n \rightarrow \infty} \frac{1}{n}\left|\left\{k \leq n: \mid x_{k}-L \geq \varepsilon\right\}\right|=0 .
$$

In symbol, we will write $S-\lim x=L$. We remark that every convergent sequence is statistically convergent but not conversely.

Let $X$ and $Y$ be two sequence spaces and let $A=\left(a_{n, k}\right)$ be an infinite matrix. If for each $x=\left(x_{k}\right)$ in $X$ the series

2010 Mathematics Subject Classification. Primary: 40G05,40G15

Key words and phrases. A-statistical convergence, nonnegative regular matrix 


$$
A_{n} x=\sum_{k} a_{n, k} x_{k}=\sum_{k=1}^{\infty} a_{n, k} x_{k}
$$

converges for each $n \in N$ and the sequence $A x=A_{n} x$ belongs to $Y$, then we say the matrix $A$ maps $X$ to $Y$. By the symbol $(X, Y)$ we denote the set of all matrices which map $X$ into $Y$.

A matrix $A$ (or a matrix map $A$ ) is called regular if $A \in(c, c)$, where the symbol $c$ denotes the spaces of all convergent sequences and

$$
\lim _{n \rightarrow \infty} A_{n} x=\lim _{k \rightarrow \infty} x_{k}
$$

for all $x \in c$. The well-known Silverman-Toeplitz theorem (see [8]) assert that $A=\left(a_{n, k}\right)$ is regular if and only if

i) $\lim _{n} a_{n, k}=0$ for each $k$;

ii) $\lim _{n} \sum_{k} a_{n, k}=1$;

iii) $\sup _{n} \sum_{k}\left|a_{n, k}\right|<\infty$.

Kolk [9] extended the definition of statistical convergence which the help of nonnegative regular matrix $A=\left(a_{n, k}\right)$ calling it $A$-statistical convergence. The definition of $A$-statistical convergence is given by Kolk as follows. For any nonnegative regular matrix $A$, we say that a sequence is $A$-statistically convergent to $L$ provided that for every $\varepsilon>0$ we have

$$
\lim _{n \rightarrow \infty} \sum_{k:\left|x_{k}-L\right| \geq \varepsilon} a_{n, k}=L
$$

In 2009, the concept of weighted statistical convergence was defined and studied by Karakaya and Chishti [10] and further modified by Mursaleen et al. [11] in 2012. In 2013, Belen and Mohiuddine [12] presented a generalization of this notion through de la Vallee-Poussin mean in probabilistic normed spaces.

Let $\sum_{k=0}^{n} x_{n}$ be a given infinite series with sequence of its $n^{\text {th }}$ partial sum $\left\{S_{n}\right\}$. If $(E, 1)$ transform is defined as

$$
E_{n}^{1}=\frac{1}{2^{n}} \sum_{k=0}^{n}\left(\begin{array}{l}
n \\
k
\end{array}\right) S_{k}
$$

and we say that this summability method is convergent if $E_{n}^{1} \rightarrow S$ as $n \rightarrow \infty$. In this case we say the series $\sum_{k=0}^{n} x_{n}$ is $(E, 1)$ - summable to a definite number S. (Hardy [31]). And we will write $S_{n} \rightarrow S(E, 1)$ as $n \rightarrow \infty$.

Let $\left(p_{n}\right)$ and $\left(q_{n}\right)$ be the two sequences of non-zero real constants such that 


$$
\begin{aligned}
& P_{n}=p_{0}+p_{1}+\ldots+p_{n}, P_{-1}=p_{-1}=0 \\
& Q_{n}=q_{0}+q_{1}+\ldots+q_{n}, Q_{-1}=q_{-1}=0
\end{aligned}
$$

For the given sequences $\left(p_{n}\right)$ and $\left(q_{n}\right)$, convolution $p^{*} q$ is defined by:

$$
R_{n}=p^{*} q=\sum_{k=0}^{n} p_{n} q_{n-k}
$$

The series $\sum_{k=0}^{n} x_{n}$ or the sequence $\left\{S_{n}\right\}$ is summable to $S$ by generalized Norlund method and it is denoted by $S_{n} \rightarrow S(N, p, q)$ if

$$
t_{n}^{p, q}=\frac{1}{R_{n}} \sum_{v=0}^{n} p_{n-v} q_{v} S_{v}
$$

tends to $S$ as $n \rightarrow \infty$.

Let us use in consideration the following method of summability:

$$
t_{n}^{p, q, E}=\frac{1}{R_{n}} \sum_{k=0}^{n} p_{n-k} q_{k} E_{k}^{1}=\frac{1}{R_{n}} \sum_{k=0}^{n} p_{n-k} q_{k} \frac{1}{2^{k}} \sum_{v=0}^{k}\left(\begin{array}{l}
k \\
v
\end{array}\right) S_{v}
$$

If $t_{n}^{p, q, E} \rightarrow S$ as $n \rightarrow \infty$, then we say that the series $\sum_{k=0}^{n} x_{n}$ or the sequence $\left\{S_{n}\right\}$ is summable to $S$ by Norlund-Euler method and it is denoted by $S_{n} \rightarrow S(N, p, q)(E, 1)$.

Remark 1. If $p_{k}=1, q_{k}=1$, then we get Euler summability method.

Now we are able to give the definition of the weighted statistical convergence related to the $(N, p, q)(E, 1)$ - summability method.

We say that $E$ have weighted density, denoted by $\delta_{N E}(E)$, if

$$
\delta_{N E}(E)=\lim _{n \rightarrow \infty} \frac{1}{R_{n}}\left|\left\{k \leq R_{n}: k \in E\right\}\right| .
$$

A sequence $x=\left(x_{k}\right)$ is said to be weighted Norlund-Euler statistical convergent (or $S_{N E}$-convergent) if for every $\varepsilon>0$ :

$$
\lim _{n \rightarrow \infty} \frac{1}{R_{n}}\left|\left\{k \leq R_{n}: p_{n-k} q_{k} \frac{1}{2^{k}} \sum_{v=0}^{k}\left(\begin{array}{l}
k \\
v
\end{array}\right) \mid x_{v}-L \geq \varepsilon\right\}\right|=0
$$

In these case we write $L=S_{N E}(s t)-\lim x$.

In the other hand, let us recall that $C[a, b]$ is the space of all functions $f$ continuous on $[a, b]$. We know that $f \in C[a, b]$ is Banach spaces with norm

$$
\|f\|_{\infty}=\sup _{x \in[a, b]}|f(x)|, f \in C[a, b]
$$


Suppose that $L$ is a linear operator from $C[a, b]$ into $C[a, b]$. It is clear that if $f \geq 0$ implies $L f \geq 0$, then the linear operator $L$ is positive on $C[a, b]$. We denote the value of $L f$ at a point $x \in[a, b]$ by $L(f ; x)$. The classical Korovkin approximation theorem states the following [14].

Theorem 2. Let $\left(T_{n}\right)$ be a sequence of positive linear operators from $C[a, b]$ into $C[a, b]$. Then,

$$
\lim _{n \rightarrow \infty}\left\|T_{n}(f ; x)-f(x)\right\|_{\infty}=0
$$

for all $C[a, b]$ if only if

$$
\lim _{n \rightarrow \infty}\left\|T_{n}\left(f_{i} ; x\right)-f_{i}(x)\right\|_{\infty}=0
$$

where $f_{i}(x)=x^{i}$ and $i=0,1,2$.

Many mathematicians extended the Korovkin-type approximation theorems by using various test functions in several setups, including Banach spaces, abstract Banach lattices, function spaces, and Banach algebras. Firstly, Gadjiev and Orhan [15] established classical Korovkin theorem through statistical convergence and display an interesting example in support of our result. Recently, Korovkin-type theorems have been obtained by Mohiuddine [16] for almost convergence. Korovkin-type theorems were also obtained in [17] for $\lambda$-statistical convergence. The authors of [18] established these types of approximation theorem in weighted $L_{p}$ spaces, where $1 \leq p<\infty$, through $A$-summability which is stronger than ordinary convergence. For these types of approximation theorems and related concepts, one can be referred to [1929] and references therein.

\section{KOROVKIN-TYPE THEOREMS BY WEIGHTED NORLUND-EULER A-STATISTiCAl CONVERGENCE}

Kolk [9] introduced the notion of $A$-statistical convergence by considering nonnegative regular matrix $A$ instead of Cesáro matrix in the definition of statistical convergence due to Fast. Inspired from the work of S. A. Mohiuddine, Abdullah Alotaibi, and Bipan Hazarika [30] we introduce the notion of weighted Norlund-Euler $A$-statistical convergence of a sequence and then we establish some Korovkin-type theorems by using this notion.

Definition 3. Let $A=\left(a_{n, k}\right)$ be a nonnegative regular matrix. A sequence $x=\left(x_{k}\right)$ of real or complex numbers is said to be weighted Norlund-Euler $A$-statistical convergence, denoted by $S_{A}^{N E}$ - convergent, to $L$ if for every $\varepsilon>0$ 


$$
\lim _{n \rightarrow \infty} \sum_{k \in E(p, \varepsilon)} a_{n, k}=0
$$

where

$$
E(p, \varepsilon)=\left\{k \in N: p_{n-k} q_{k} \frac{1}{2^{k}} \sum_{v=0}^{k}\left(\begin{array}{l}
k \\
v
\end{array}\right)\left|x_{v}-L\right| \geq \varepsilon\right\}
$$

In symbol, we will write $S_{A}^{N E}-\lim x=L$.

Remark 4. Note that convergence sequence implies weighted Norlund-Euler $A$ statistical convergent to the same value but converse is not true in general. For example, take $p_{k}=1, q_{k}=1$ for all $k$ and define a sequence $x=\left(x_{k}\right)$ by

$$
x_{k}= \begin{cases}1, & \text { if } k=n^{2} \\ 0, & \text { otherwise }\end{cases}
$$

where $n \in N$. Then this sequence is statistically convergent to 0 but not convergent; in this case, weighted Norlund-Euler $A$-statistical convergence of a sequence coincides with statistical convergence.

Theorem 5. Let $A=\left(a_{n, k}\right)$ be a nonnegative regular matrix. Consider a sequence of positive linear operators $\left(M_{k}\right)$ from $C[a, b]$ into itself. Then, for all $f \in C[a, b]$ bounded on whole real line,

$$
S_{A}^{N E}-\lim _{k \rightarrow \infty}\left\|M_{k}(f ; x)-f(x)\right\|_{\infty}=0
$$

if only if

$$
\begin{aligned}
& S_{A}^{N E}-\lim _{k \rightarrow \infty}\left\|M_{k}(1 ; x)-1\right\|_{\infty}=0, \\
& S_{A}^{N E}-\lim _{k \rightarrow \infty} M_{k}\|(v ; x)-x\|_{\infty}=0, \\
& S_{A}^{N E}-\lim _{k \rightarrow \infty}\left\|M_{k}\left(v^{2} ; x\right)-x^{2}\right\|_{\infty}=0
\end{aligned}
$$

Proof. Equation (20) directly follows from (19) because each of $1, x, x^{2}$ belongs to $C[a, b]$. Consider a function $f \in C[a, b]$. Then there is a constant $C>0$ such that $|f(x)| \leq C$ for all $x \in(-\infty,+\infty)$. Therefore,

$$
|f(v)-f(x)| \leq 2 C, \quad-\infty<v, x<+\infty,
$$

Let $\varepsilon>0$ be given. By hypothesis there is a $\delta=\delta(\varepsilon)>0$ such that

$$
|f(v)-f(x)|<\varepsilon, \quad \forall|v-x|<\delta
$$

Solving (21) and (22) and then substituting $\Omega(v)=(v-x)^{2}$, one obtains

$$
|f(v)-f(x)|<\varepsilon+\frac{2 C}{\delta^{2}} \Omega .
$$

Equation (23) can be also written by as 


$$
-\varepsilon-\frac{2 C}{\delta^{2}} \Omega<f(v)-f(x)<\varepsilon+\frac{2 C}{\delta^{2}} \Omega .
$$

Operating $M_{k}(1 ; x)$ to $(24)$ since $M_{k}(f ; x)$ his linear and monoton, one obtains

$$
M_{k}(1 ; x)\left(-\varepsilon-\frac{2 C}{\delta^{2}} \Omega\right)<M_{k}(1 ; x)(f(v)-f(x))<M_{k}(1 ; x)\left(\varepsilon+\frac{2 C}{\delta^{2}} \Omega\right)
$$

Note that $x$ is fixed, so $f(x)$ is constant number. Thus, we obtain from (25) that

$$
-\varepsilon M_{k}(1 ; x)-\frac{2 C}{\delta^{2}} M_{k}(\Omega ; x)<M_{k}(f ; x)-f(x) M_{k}(1 ; x)<\varepsilon M_{k}(1 ; x)+\frac{2 C}{\delta^{2}} M_{k}(\Omega ; x)
$$

The term " $M_{k}(f ; x)-f(x) M_{k}(1 ; x) "$ in (26) can also written as

$$
M_{k}(f ; x)-f(x) M_{k}(1 ; x)=M_{k}(f ; x)-f(x)-f(x)\left[M_{k}(1 ; x)-1\right]
$$

Now substituting the value of $M_{k}(f ; x)-f(x) M_{k}(1 ; x)$ in (26), we get that

$$
M_{k}(f ; x)-f(x)<\varepsilon M_{k}(1 ; x)+\frac{2 C}{\delta^{2}} M_{k}(\Omega ; x)+f(x)\left[M_{k}(1 ; x)-1\right]
$$

We can rewrite the term " $M_{k}(\Omega ; x)$ " in (28) as follows:

$$
\begin{aligned}
M_{k}(\Omega ; x) & =M_{k}\left((v-x)^{2} ; x\right)=M_{k}\left(v^{2} ; x\right)+2 x M_{k}(v ; x)+x^{2} M_{k}(1 ; x) \\
& =\left[M_{k}\left(v^{2} ; x\right)-x^{2}\right]-2 x\left[M_{k}(v ; x)-x\right]+x^{2}\left[M_{k}(1 ; x)-1\right]
\end{aligned}
$$

Equation (28) with the above value of $M_{k}(\Omega ; x)$ becomes

$$
\begin{aligned}
M_{k}(f ; x)-f(x)< & \varepsilon M_{k}(1 ; x)+\frac{2 C}{\delta^{2}}\left\{\left[M_{k}\left(v^{2} ; x\right)-x^{2}\right]+2 x\left[M_{k}(v ; x)-x\right]\right. \\
& \left.+x^{2}\left[M_{k}(1 ; x)-1\right]\right\}+f(x)\left[M_{k}(1 ; x)-1\right] \\
= & \varepsilon\left[M_{k}(1 ; x)-1\right]+\varepsilon+\frac{2 C}{\delta^{2}}\left\{\left[M_{k}\left(v^{2} ; x\right)-x^{2}\right]+2 x\left[M_{k}(v ; x)-x\right]\right. \\
& \left.+x^{2}\left[M_{k}(1 ; x)-1\right]\right\}+f(x)\left[M_{k}(1 ; x)-1\right]
\end{aligned}
$$

Therefore,

$$
\begin{aligned}
\left|M_{k}(f ; x)-f(x)\right| \leq & \left(\varepsilon+\frac{2 C b^{2}}{\delta^{2}}+C\right)\left|M_{k}(1 ; x)-1\right| \\
& +\frac{2 C}{\delta^{2}}\left|M_{k}\left(v^{2} ; x\right)-x^{2}\right| \\
& +\frac{4 C b}{\delta^{2}}\left|M_{k}(v ; x)-x\right|
\end{aligned}
$$

where $b=\max |x|$. Taking supremum over $x \in[a, b]$, one obtains

$$
\begin{aligned}
\left\|M_{k}(f ; x)-f(x)\right\|_{\infty} \leq & \left(\varepsilon+\frac{2 C b^{2}}{\delta^{2}}+C\right)\left\|M_{k}(1 ; x)-1\right\|_{\infty} \\
& +\frac{2 C}{\delta^{2}}\left\|M_{k}\left(v^{2} ; x\right)-x^{2}\right\|_{\infty} \\
& +\frac{4 C b}{\delta^{2}}\left\|M_{k}(v ; x)-x\right\|_{\infty}
\end{aligned}
$$

or

$$
\begin{aligned}
\left\|M_{k}(f ; x)-f(x)\right\|_{\infty} \leq & T\left\{\left\|M_{k}(1 ; x)-1\right\|_{\infty}\right. \\
& +\left\|M_{k}\left(v^{2} ; x\right)-x^{2}\right\|_{\infty} \\
& \left.+\left\|M_{k}(v ; x)-x\right\|_{\infty}\right\}
\end{aligned}
$$


where

$$
T=\max \left\{\varepsilon+\frac{2 C b^{2}}{\delta^{2}}+C, \frac{2 C}{\delta^{2}}, \frac{4 C b}{\delta^{2}}\right\}
$$

Hence

$$
\begin{aligned}
p_{n-k} q_{k} \frac{1}{2^{k}} \sum_{v=0}^{k}\left(\begin{array}{l}
k \\
v
\end{array}\right)\left\|M_{k}(f ; x)-f(x)\right\|_{\infty} \leq & T\left\{p_{n-k} q_{k} \frac{1}{2^{k}} \sum_{v=0}^{k}\left(\begin{array}{l}
k \\
v
\end{array}\right)\left\|M_{k}(1 ; x)-1\right\|_{\infty}\right. \\
& +p_{n-k} q_{k} \frac{1}{2^{k}} \sum_{v=0}^{k}\left(\begin{array}{l}
k \\
v
\end{array}\right)\left\|M_{k}\left(v^{2} ; x\right)-x^{2}\right\|_{\infty} \\
& \left.+p_{n-k} q_{k} \frac{1}{2^{k}} \sum_{v=0}^{k}\left(\begin{array}{l}
k \\
v
\end{array}\right)\left\|M_{k}(v ; x)-x\right\|_{\infty}\right\}
\end{aligned}
$$

For given $\alpha>0$, choose $\varepsilon>0$ such that $\varepsilon<\alpha$, and we will define the following sets:

$$
\begin{aligned}
& E=\left\{k \in N: p_{n-k} q_{k} \frac{1}{2^{k}} \sum_{v=0}^{k}\left(\begin{array}{l}
k \\
v
\end{array}\right)\left\|M_{k}(f ; x)-f(x)\right\|_{\infty} \geq \alpha\right\} \\
& E_{1}=\left\{k \in N: p_{n-k} q_{k} \frac{1}{2^{k}} \sum_{v=0}^{k}\left(\begin{array}{l}
k \\
v
\end{array}\right)\left\|M_{k}(1, x)-1\right\|_{\infty} \geq \frac{\alpha-\varepsilon}{3 T}\right\} \\
& E_{2}=\left\{k \in N: p_{n-k} q_{k} \frac{1}{2^{k}} \sum_{v=0}^{k}\left(\begin{array}{l}
k \\
v
\end{array}\right)\left\|M_{k}(v ; x)-x\right\|_{\infty} \geq \frac{\alpha-\varepsilon}{3 T}\right\} \\
& E_{3}=\left\{k \in N: p_{n-k} q_{k} \frac{1}{2^{k}} \sum_{v=0}^{k}\left(\begin{array}{l}
k \\
v
\end{array}\right)\left\|M_{k}\left(v^{2} ; x\right)-x^{2}\right\|_{\infty} \geq \frac{\alpha-\varepsilon}{3 T}\right\}
\end{aligned}
$$

It easy to see that

$$
E \subset E_{1} \cup E_{2} \cup E_{3}
$$

Thus, for each $n \in N$, we obtain from (35) that

$$
\sum_{k \in E} a_{n, k} \leq \sum_{k \in E_{1}} a_{n, k}+\sum_{k \in E_{2}} a_{n, k}+\sum_{k \in E_{3}} a_{n, k}
$$

Taking limit $n \rightarrow \infty$ in (38) and also (20) gives that

$$
\lim _{n \rightarrow \infty} \sum_{k \in E} a_{n, k}=0 \text {. }
$$

These yields that

$$
S_{A}^{N E}-\lim _{k \rightarrow \infty}\left\|M_{k}(f ; x)-f(x)\right\|_{\infty}=0
$$

for all $f \in C[a, b]$.

We also obtain the following Korovkin-type theorem for weighted Norlund-Euler statistical convergence instead of nonnegative regular matrix $A$ in Theorem 5.

Theorem 6. Consider a sequence of positive linear operators $\left(M_{k}\right)$ from $C[a, b]$ into itself. Then, for all $f \in C[a, b]$ bounded on whole real line,

$$
S_{N E}-\lim _{k \rightarrow \infty}\left\|M_{k}(f ; x)-f(x)\right\|_{\infty}=0
$$


if only if

$$
\begin{aligned}
& S_{N E}-\lim _{k \rightarrow \infty}\left\|M_{k}(1 ; x)-1\right\|_{\infty}=0 \\
& S_{N E}-\lim _{k \rightarrow \infty}\left\|M_{k}(v ; x)-x\right\|_{\infty}=0 \\
& S_{N E}-\lim _{k \rightarrow \infty}\left\|M_{k}\left(v^{2} ; x\right)-x^{2}\right\|_{\infty}=0
\end{aligned}
$$

Proof. Following the proof of Theorem 5, one obtains

$$
E \subset E_{1} \cup E_{2} \cup E_{3}
$$

and so

$$
\delta_{N E}(E) \subset \delta_{N E}\left(E_{1}\right)+\delta_{N E}\left(E_{2}\right)+\delta_{N E}\left(E_{3}\right)
$$

Equations (42)-(44) give that

$$
S_{N E}-\lim _{k \rightarrow \infty}\left\|M_{k}(f ; x)-f(x)\right\|_{\infty}=0 .
$$

Remark 7. By the Theorem 2 of [32], we have that if a sequence $x=\left(x_{k}\right)$ is weighted Norlun-Euler statistically convergent to $L$, then it is strongly $(N, p, q)(E, 1)-$ summable to $L$, provided that $p_{n-k} q_{k} \frac{1}{2^{k}} \sum_{v=0}^{k}\left(\begin{array}{l}k \\ v\end{array}\right)\left|x_{k}-L\right|$ is bounded; that is, there exist a constant $C$ such that

$$
p_{n-k} q_{k} \frac{1}{2^{k}} \sum_{v=0}^{k}\left(\begin{array}{l}
k \\
v
\end{array}\right)\left|x_{k}-L\right| \leq C
$$

for all $k \in N$. We write

$$
|(N, p, q)(E, 1)|=\left\{x=x_{n}: \lim _{n \rightarrow \infty} \frac{1}{R_{n}} \sum_{k=0}^{n} p_{n-k} q_{k} \frac{1}{2^{k}} \sum_{v=0}^{k}\left(\begin{array}{l}
k \\
v
\end{array}\right)\left|x_{v}-L\right|=0 \text { for some } L\right\}
$$

the set of all sequences $x=\left(x_{k}\right)$ which are strongly $(N, p, q)(E, 1)-$ summable to $L$.

Theorem 8. Let $M_{k}: C[a, b] \rightarrow C[a, b]$ be a sequence of positive linear operators which satisfies (43)-(44) of Theorem 6 and the following conditions holds:

$$
\lim _{k \rightarrow \infty}\left\|M_{k}(1 ; x)-1\right\|_{\infty}=0 \text {. }
$$

Then,

$$
\lim _{\mathrm{n} \rightarrow \infty} \frac{1}{R_{n}} \sum_{k=0}^{n} p_{n-k} q_{k} \frac{1}{2^{k}} \sum_{v=0}^{k}\left(\begin{array}{l}
k \\
v
\end{array}\right)\left\|M_{k}(f ; x)-f(x)\right\|_{\infty}=0,
$$

for any $f \in C[a, b]$.

Proof. It follow from (49) that $\left\|M_{k}(f ; x)\right\|_{\infty} \leq C^{\prime}$, for some constant $C^{\prime}>0$ and for all $k \in N$. Hence for $f \in C[a, b]$, one obtains 


$$
\begin{aligned}
p_{n-k} q_{k} \frac{1}{2^{k}} \sum_{v=0}^{k}\left(\begin{array}{l}
k \\
v
\end{array}\right)\left\|M_{k}(f ; x)-f(x)\right\|_{\infty} & \leq p_{n-k} q_{k} \frac{1}{2^{k}} \sum_{v=0}^{k}\left(\begin{array}{l}
k \\
v
\end{array}\right)\left(\|f\|_{\infty}\left\|M_{k}(1 ; x)\right\|_{\infty}+\|f\|_{\infty}\right) \\
& \leq p_{n-k} q_{k} \frac{1}{2^{k}} \sum_{v=0}^{k}\left(\begin{array}{l}
k \\
v
\end{array}\right) C\left(C^{\prime}+1\right) .
\end{aligned}
$$

Right hand side of (51) is constant, so

$$
p_{n-k} q_{k} \frac{1}{2^{k}} \sum_{v=0}^{k}\left(\begin{array}{l}
k \\
v
\end{array}\right)\left\|M_{k}(f ; x)-f(x)\right\|_{\infty}
$$

is bounded. Since (49) implies (42), by Theorem 6 we get that

$$
S_{N E}-\lim _{k \rightarrow \infty}\left\|M_{k}(f ; x)-f(x)\right\|_{\infty}=0 .
$$

By remark 7, (51) and (52) together give the desired result.

\section{RATE OF Weighted NORLUND-EULER A-STATISTICAL CONVERGENCE}

First we define the rate of weighted Norlund-Euler A-statistical convergent sequence as follows.

Definition 9. Let $A=\left(a_{n, k}\right)$ be a nonnegative regular matrix and let $\left(a_{k}\right)$ be a positive non increasing sequence. Then, a sequence $x=\left(x_{k}\right)$ is weighted Norlund-Euler Astatistical convergent to $L$ with the rate of $o\left(a_{k}\right)$ if for each $\varepsilon>0$

$$
\lim _{n \rightarrow \infty} \frac{1}{a_{n}} \sum_{k \in E(p, \varepsilon)} a_{n, k}=0
$$

where

$$
E(p, \varepsilon)=\left\{k \in N: p_{n-k} q_{k} \frac{1}{2^{k}} \sum_{v=0}^{k}\left(\begin{array}{l}
k \\
v
\end{array}\right)\left|x_{v}-L\right| \geq \varepsilon\right\}
$$

In symbol, we will write

$$
x_{k}-L=S_{A}^{N E}-o\left(a_{k}\right) \text { as } k \rightarrow \infty
$$

We will prove the following auxiliary result by using the above definition.

Lemma 10. Let $A=\left(a_{n, k}\right)$ be a nonnegative regular matrix. Suppose that $\left(a_{k}\right)$ and $\left(b_{k}\right)$ are two positive nonincreasing sequences. Let $x=\left(x_{k}\right)$ and $y=\left(y_{k}\right)$ be two sequences such that

$$
x_{k}-L_{1}=S_{A}^{N E}-o\left(a_{k}\right) \text { and } y_{k}-L_{2}=S_{A}^{N E}-o\left(b_{k}\right) .
$$

Then,

(i) $\left(x_{k}-L_{1}\right) \pm\left(y_{k}-L_{2}\right)=S_{A}^{N E}-o\left(c_{k}\right)$,

(ii) $\left(x_{k}-L_{1}\right)\left(y_{k}-L_{2}\right)=S_{A}^{N E}-o\left(a_{k} b_{k}\right)$, 
(iii) $\alpha\left(x_{k}-L_{1}\right)=S_{A}^{N E}-o\left(a_{k}\right)$, for any scalar $\alpha$,

where $c_{k}=\max \left\{a_{k}, b_{k}\right\}$.

Proof. Suppose that

$$
x_{k}-L_{1}=S_{A}^{N E}-o\left(a_{k}\right), y_{k}-L_{2}=S_{A}^{N E}-o\left(b_{k}\right)
$$

Given $\varepsilon>0$, define

$$
\begin{aligned}
& E^{\prime}=\left\{k \in N: p_{n-k} q_{k} \frac{1}{2^{k}} \sum_{v=0}^{k}\left(\begin{array}{l}
k \\
v
\end{array}\right)\left|\left(x_{k}-L_{1}\right) \pm\left(y_{k}-L_{2}\right)\right| \geq \varepsilon\right\} \\
& E^{\prime \prime}=\left\{k \in N: p_{n-k} q_{k} \frac{1}{2^{k}} \sum_{v=0}^{k}\left(\begin{array}{l}
k \\
v
\end{array}\right)\left|x_{k}-L_{1}\right| \geq \frac{\varepsilon}{2}\right\} \\
& E^{\prime \prime \prime}=\left\{k \in N: p_{n-k} q_{k} \frac{1}{2^{k}} \sum_{v=0}^{k}\left(\begin{array}{l}
k \\
v
\end{array}\right)\left|y_{k}-L_{2}\right| \geq \frac{\varepsilon}{2}\right\}
\end{aligned}
$$

It easy to see that

$$
E^{\prime} \subset E^{\prime \prime} \cup E^{\prime \prime}
$$

These yields that

$$
\frac{1}{c_{n}} \sum_{k \in E^{\prime}} a_{n, k} \leq \frac{1}{c_{n}} \sum_{k \in E^{\prime \prime}} a_{n, k}+\frac{1}{c_{n}} \sum_{k \in E^{\prime \prime}} a_{n, k}
$$

holds for all $n \in N$. Since $c_{k}=\max \left\{a_{k}, b_{k}\right\}$, (59) gives that

$$
\frac{1}{c_{n}} \sum_{k \in E^{\prime}} a_{n, k} \leq \frac{1}{a_{n}} \sum_{k \in E^{\prime \prime}} a_{n, k}+\frac{1}{b_{n}} \sum_{k \in E^{\prime \prime \prime}} a_{n, k}
$$

Taking limit $n \rightarrow \infty$ in (60) together with (56), we obtain

$$
\lim _{n \rightarrow \infty} \frac{1}{c_{n}} \sum_{k \in E^{\prime}} a_{n, k}=0
$$

Thus,

$$
\left(x_{k}-L_{1}\right) \pm\left(y_{k}-L_{2}\right)=S_{A}^{N E}-o\left(c_{k}\right)
$$

Similarly, we can prove (ii) and (iii).

Now, we recall the notion of modulus of continuity of $f$ in $C[a, b]$ is defined by

$$
\omega(f, \delta)=\sup \{|f(x)-f(y)|: x, y \in[a, b],|x-y|<\delta\}
$$

It is well known that

$$
|f(x)-f(y)| \leq \omega(f, \delta)\left(\frac{|x-y|}{\delta}+1\right) .
$$

Theorem 11. Let $A=\left(a_{n, k}\right)$ be a nonnegative regular matrix. If the sequence of positive linear operators $M_{k}: C[a, b] \rightarrow C[a, b]$ satisfies the conditions

(i) $\left\|M_{k}(1 ; x)-1\right\|_{\infty}=S_{A}^{N E}-o\left(a_{k}\right)$,

(ii) $\omega\left(f, \lambda_{k}\right)=S_{A}^{N E}-o\left(b_{k}\right)$, with $\lambda_{k}=\sqrt{M_{k}\left(\varphi_{x} ; x\right)}$ and $\varphi_{x}(y)=(y-x)^{2}$, 
where $\left(a_{k}\right)$ and $\left(b_{k}\right)$ are two positive nonincreasing sequences, then

$$
\left\|M_{k}(f ; x)-f(x)\right\|_{\infty}=S_{A}^{N E}-o\left(c_{k}\right)
$$

for all $f \in C[a, b]$, where $c_{k}=\max \left\{a_{k}, b_{k}\right\}$.

Proof. Equation (27) can be reformed into the following form:

$$
\begin{aligned}
& \left|M_{k}(f ; x)-f(x)\right| \leq M_{k}(|f(x)-f(y)| ; x)+|f(x)| \cdot\left|M_{k}(1 ; x)-1\right| \\
& \quad \leq M_{k}\left(1+\frac{|y-x|}{\delta} ; x\right) \omega(f, \delta)+|f(x)| \cdot\left|M_{k}(1 ; x)-1\right| \\
& \quad \leq M_{k}\left(1+\frac{(y-x)^{2}}{\delta^{2}} ; x\right) \omega(f, \delta)+|f(x)| \cdot\left|M_{k}(1 ; x)-1\right| \\
& \quad \leq\left(M_{k}(1 ; x)+\frac{1}{\delta^{2}} M_{k}\left(\varphi_{x} ; x\right)\right) \omega(f, \delta)+|f(x)| \cdot\left|M_{k}(1 ; x)-1\right| \\
& \quad \leq\left|M_{k}(1 ; x)-1\right| \omega(f, \delta)+|f(x)| \cdot\left|M_{k}(1 ; x)-1\right|+\omega(f, \delta)+\frac{1}{\delta^{2}} M_{k}\left(\varphi_{x} ; x\right) \omega(f, \delta)
\end{aligned}
$$

Choosing $\delta=\lambda_{k}=\sqrt{M_{k}\left(\varphi_{x} ; x\right)}$, one obtains

$$
\left\|M_{k}(f ; x)-f(x)\right\|_{\infty} \leq T\left\|M_{k}(1 ; x)-1\right\|_{\infty}+2 \omega\left(f, \lambda_{k}\right)+\left\|M_{k}(1 ; x)-1\right\|_{\infty} \omega\left(f, \lambda_{k}\right)
$$

where $T=\|f\|_{\infty}$. For given $\varepsilon>0$, we will define the following sets:

$$
\begin{aligned}
& E_{1}^{\prime}=\left\{k \in N: p_{n-k} q_{k} \frac{1}{2^{k}} \sum_{v=0}^{k}\left(\begin{array}{l}
k \\
v
\end{array}\right)\left\|M_{k}(f ; x)-f(x)\right\|_{\infty} \geq \varepsilon\right\} \\
& E_{2}^{\prime}=\left\{k \in N: p_{n-k} q_{k} \frac{1}{2^{k}} \sum_{v=0}^{k}\left(\begin{array}{l}
k \\
v
\end{array}\right)\left\|M_{k}(1, x)-1\right\|_{\infty} \geq \frac{\varepsilon}{3 T}\right\} \\
& E_{3}^{\prime}=\left\{k \in N: p_{n-k} q_{k} \frac{1}{2^{k}} \sum_{v=0}^{k}\left(\begin{array}{l}
k \\
v
\end{array}\right) \omega\left(f, \lambda_{k}\right) \geq \frac{\varepsilon}{6}\right\} \\
& E_{4}^{\prime}=\left\{k \in N: p_{n-k} q_{k} \frac{1}{2^{k}} \sum_{v=0}^{k}\left(\begin{array}{l}
k \\
v
\end{array}\right) \omega\left(f, \lambda_{k}\right)\left\|M_{k}(1 ; x)-1\right\|_{\infty} \geq \frac{\varepsilon}{3}\right\} .
\end{aligned}
$$

It follow from (67) that

$$
\frac{1}{c_{n}} \sum_{k \in E_{1}^{\prime}} a_{n, k} \leq \frac{1}{c_{n}} \sum_{k \in E_{2}^{\prime}} a_{n, k}+\frac{1}{c_{n}} \sum_{k \in E_{3}^{\prime}} a_{n, k}+\frac{1}{c_{n}} \sum_{k \in E_{4}^{\prime}} a_{n, k}
$$

holds for $n \in N$. Since $c_{k}=\max \left\{a_{k}, b_{k}\right\}$, we obtain from (69) that

$$
\frac{1}{c_{n}} \sum_{k \in E_{1}^{\prime}} a_{n, k} \leq \frac{1}{a_{n}} \sum_{k \in E_{2}^{\prime}} a_{n, k}+\frac{1}{b_{n}} \sum_{k \in E_{3}^{\prime}} a_{n, k}+\frac{1}{c_{n}} \sum_{k \in E_{4}^{\prime}} a_{n, k} .
$$

Taking limit $n \rightarrow \infty$ in (70) together with Lemma 10 and our hypotheses (i) and (ii), one obtains

$$
\lim _{n \rightarrow \infty} \frac{1}{c_{n}} \sum_{k \in E_{1}^{\prime}} a_{n, k}=0
$$

These yields

$$
\left\|M_{k}(f ; x)-f(x)\right\|_{\infty}=S_{A}^{N E}-o\left(c_{k}\right)
$$




\section{References}

[1] H. Fast, Sur la convergence statistique, Colloquium Mathematicum,vol. 2, pp. 241-244, 1951.

[2] A. Zygmund, Trigonometric Series, vol. 5 of Monografje Matematyczne, WarszawaLwow, 1935.

[3] A. Zygmund, Trigonometric Series, CambridgeUniversity Press,Cambridge, UK, 1959.

[4] I. J. Schoenberg, The integrability of certain functions and related summability methods," The American Mathematical Monthly, vol. 66, pp. 361-375, 1959.

[5] T. Šalát, On statistically convergent sequences of real numbers, Mathematica Slovaca, vol. 30, no. 2, pp. 139-150, 1980.

[6] J. A. Fridy, On statistical convergence, Analysis, vol. 5, no. 4, pp. 301-313, 1985.

[7] J. S. Connor, The statistical and strong $p$-Cesaro convergence of sequences, Analysis, vol. 8, no. 1-2, pp. 47-63, 1988.

[8] R. G. Cooke, Infinite Matrices and Sequence Spaces, Macmillan,London, UK, 1950.

[9] E. Kolk, Matrix summability of statistically convergent sequences, Analysis, vol. 13, no. 12, pp. 77-83, 1993.

[10] V. Karakaya, T. A. Chishti, Weighted statistical convergence, Iranian Journal of Science and Technology A, vol. 33, no.3, pp. 219-223, 2009.

[11] M. Mursaleen, V. Karakaya, M. Ertürk, F. Gürsoy, Weighted statistical convergence and its application to Korovkin type approximation theorem, Applied Mathematics and Computation, vol. 218, no. 18, pp. 9132-9137, 2012.

[12] C. Belen, S. A. Mohiuddine, Generalized weighted statistical convergence and application, Applied Mathematics and Computation, vol. 219, no. 18, pp. 9821-9826, 2013.

[13] A. Esi, Statistical summability through de la Vallée-Poussin mean in probabilistic normed space, International Journal of Mathematics and Mathematical Sciences, vol. 2014, Article ID 674159, 5 pages, 2014.

[14] P. P. Korovkin, Linear Operators and Approximation Theory, Hindustan Publishing, New Delhi, India, 1960.

[15] A. D. Gadjiev and C. Orhan, Some approximation theorems via statistical convergence, The Rocky Mountain Journal of Mathematics, vol. 32, no. 1, pp. 129-138, 2002.

[16] S. A. Mohiuddine, An application of almost convergence in approximation theorems, Applied Mathematics Letters, vol. 24, no. 11, pp. 1856-1860, 2011.

[17] O. H. H. Edely, S. A. Mohiuddine, A. Noman, Korovkin type approximation theorems obtained through generalized statistical convergence, Applied Mathematics Letters, vol. 23,no. 11, pp. 1382-1387, 2010.

[18] T. Acar, F. Dirik, Korovkin-type theorems in weighted $L^{p}$-spaces via summation process, The Scientific World Journal, vol.2013, Article ID 534054, 6 pages, 2013.

[19] N. L. Braha, H. M. Srivastava, S. A. Mohiuddine, A Korovkin's type approximation theorem for periodic functions via the statistical summability of the generalized de la Vallée Poussin mean, Applied Mathematics and Computation, vol. 228, pp. 162-169, 2014.

[20] O. Duman, M. K. Khan, C. Orhan, $A-$ statistical convergence of approximating operators, Mathematical Inequalities \& Applications, vol. 6, no. 4, pp. 689-699, 2003.

[21] O. Duman, C. Orhan, Statistical approximation by positive linear operators, Studia Mathematica, vol. 161, no. 2, pp. 187-197, 2004. 
[22] M. Mursaleen, A. Kiliçman, Korovkin second theorem via B-statistical A-summability, Abstract and Applied Analysis, vol. 2013, Article ID 598963, 6 pages, 2013.

[23] S. A. Mohiuddine, A. Alotaibi, Statistical convergence and approximation theorems for functions of two variables, Journal of Computational Analysis and Applications, vol. 15, no. 2, pp.218-223, 2013.8 The ScientificWorld Journal

[24] S. A. Mohiuddine, A. Alotaibi, Korovkin second theorem via statistical summability (C, 1), Journal of Inequalities and Applications, vol. 2013, article 149, 9 pages, 2013.

[25] H. M. Srivastava, M. Mursaleen, A. Khan, Generalized equi-statistical convergence of positive linear operators and associated approximation theorems, Mathematical and Computer Modelling, vol. 55, no. 9-10, pp. 2040-2051, 2012.

[26] O. H. H. Edely, M. Mursaleen, A. Khan, Approximation for periodic functions via weighted statistical convergence, Applied Mathematics and Computation, vol. 219, no. 15, pp. 8231-8236, 2013.

[27] V. N. Mishra, K. Khatri, L. N. Mishra, Statistical approximation by Kantorovich-type discrete q-Beta operators, Advances in Difference Equations, vol. 2013, article 345, 2013.

[28] S. N. Bernstein, Démonstration du théoréme de Weierstrass fondée sur le calcul des probabilités, Communications of the Kharkov Mathematical Society, vol. 13, no. 2, pp. 1.

[29] E. A. Aljimi, E, Hoxha, V. Loku, Some Results of Weighted Norlund-Euler Statistical Convergence, International Mathematical Forum, Vol. 8, 2013, no. 37, 1797 - 1812 HIKARI Ltd.

[30] S. A. Mohiuddine, A. Alotaibi, B, Hazarika, Weighted $A$ - Statistical Convergence for Sequences of Positive Linear Operators. Hindawi Publishing Corporation, The Scientific World Journal Volume 2014, Article ID 437863, 8 pages http://dx.doi.org/10.1155/2014/437863.

[31] G. Hardy, Divergent series, first edition, Oxford University Press, 70. 1949.

[32] E. A. Aljimi, V. Loku, Generalized Weighted Norlund-Euler Statistical Convergence, Int. Journal of Math. Analysis, Vol. 8, 2014, no. 7, 345-354 HIKARI Ltd.

\footnotetext{
${ }^{1}$ Department of Mathematics, University of Tirana, Albania.

E-mail address: hoxhaelida@yahoo.com

${ }^{2}$ Department of Mathematics, University of Tirana, Albania.

E-mail address:ekremhalimii@yahoo.co.uk

${ }^{3}$ Department of Computer Sciences and Applied Mathematics,

College, Vizioni per Arsim, Ahmet Kaciku, Nr=3, Ferizaj
} 